\title{
Transesophageal echocardiography in swine: evaluation of left and right ventricular structure, function and myocardial work
}

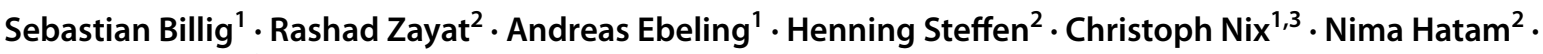 \\ Heike Schnöring ${ }^{2} \cdot$ Matthias Derwall $^{1}$ (1)
}

Received: 11 July 2020 / Accepted: 29 September 2020 / Published online: 13 October 2020

(c) The Author(s) 2020

\begin{abstract}
This study aimed to determine standard left (LV) and right ventricular (RV) transesophageal echocardiographic (TEE) measurements in swine. Additionally, global myocardial work index (GWI) was estimated using pressure-strain loops (PSL). A comprehensive TEE examination was conducted in ten anesthetized, intubated and mechanically ventilated healthy female German landrace swine, weighing 44 to $57 \mathrm{~kg}$. For GWI calculation, we performed LV and RV segmental strain analysis and used invasively measured LV and RV pressure to obtain PSL. The GWI and further myocardial work indices were calculated from the area of the PSL using commercially available software. Furthermore, hemodynamic measurements were obtained using indwelling catheters. We obtained complete standardized baseline values for left and right ventricular dimensions and function. Biplane LV ejection fraction was $63 \pm 7 \%$ and the LV end-diastolic volume was $70.5 \pm 5.9 \mathrm{ml}$. Tissue Doppler estimated peak tricuspid annular systolic velocity was $13.1 \pm 1.8 \mathrm{~cm} / \mathrm{s}$. The Doppler estimated LV and RV stroke volume index were $75.6 \pm 7.2 \mathrm{ml} / \mathrm{m}^{2}$ and $76.7 \pm 7.8 \mathrm{ml} / \mathrm{m}^{2}$ respectively. Pulsed wave Doppler derived cardiac output correlated well with cardiac output estimated using the thermodilution method $(7.0 \pm 1.2 \mathrm{l} / \mathrm{min}$ vs. $7.0 \pm 1.1 \mathrm{l} / \mathrm{min}, \mathrm{r}=0.812, \mathrm{p}=0.004)$. The LV global longitudinal strain was $-21.3 \pm 3.9 \%$ and the RV global longitudinal strain was $-15.4 \pm 2.5 \%$. LV GWI was 1885(1281-2121) $\mathrm{mmHg} * \%$ and $297 \pm 62 \mathrm{mmHg} * \%$ for the RV. LV global myocardial work efficiency was $82.6 \pm 4 \%$ and 83(72-88) \% for the RV. TEE offers sufficient morphological, functional and hemodynamic assessment of the heart in swine. Myocardial contractility and mechanics can be reliably evaluated with the non-invasive GWI derived from echocardiography without additional invasive measures.
\end{abstract}

Keywords Transesophageal echocardiography $\cdot$ Non-invasive hemodynamic monitoring $\cdot$ Speckle tracking $\cdot$ Myocardial work $\cdot$ Myocardial contractility $\cdot$ Swine model

\section{Introduction}

Sebastian Billig, Rashad Zayat, Heike Schnöring and Matthias Derwall have contributed equally to this work.

Matthias Derwall

mderwall@ukaachen.de

1 University Hospital RWTH Aachen, Department of Anesthesiology, Medical Faculty, RWTH Aachen University, Pauwelsstr. 30, Aachen 52074, Germany

2 University Hospital RWTH Aachen, Department of Thoracic and Cardiovascular Surgery, Medical Faculty, RWTH Aachen University, Pauwelsstr. 30, Aachen 52074, Germany

3 Abiomed Europe GmbH, Neuenhofer Weg 3, Aachen 52074, Germany
Echocardiography is an invaluable diagnostic tool for analysis of cardiac anatomy and function [1,2]. Therefore, it is widely used in daily clinical routine as well as in research settings. Since the porcine cardiovascular system closely resembles human anatomy and physiology, swine are frequently used as animal models [3-5]. Echocardiography has been widely used in different porcine models such as myocardial infarction [6-10]. However, transthoracic echocardiography (TTE) in swine is associated with restrictions due to the keel-shaped thorax and narrow spaced ribs [11]. For instance, TTE can be limited in yielding reliable measurements to assess systolic and diastolic function, as apical views can frequently not be obtained in closedchest swine models $[7,12,13]$. In contrast, transesophageal 
echocardiography (TEE) is free of these limitations [14, 15]. Here we report our findings regarding the use of TEE in swine. Only few publications have yet systematically described acquisition and results from porcine TEE-studies [14-16]. Moreover, data on right ventricular (RV) function and myocardial mechanics are lacking. Specifically, we aimed to determine the normal baseline values of standard two- dimensional (2D) and speckle-tracking echocardiography (2DSTE) measurements of the left ventricle (LV) and right ventricle (RV) in German landrace swine under general anesthesia. Baseline values of LV and RV dimensions, function and mechanics were determined using 2D imaging, Doppler modality, tissue Doppler and 2DSTE. Additionally, we applied a novel noninvasive method to calculate global myocardial work indices (GWI) using pressure-strain loops as described by Russel and colleagues [17-20].

\section{Methods}

The experimental protocol was approved by the appropriate governmental institution (Landesamt für Natur, Umwelt und Verbraucherschutz Nordrhein-Westfalen, LANUV NRW). All animals received adequate care according to the precepts of the Helsinki Declaration of 1964 and its later amendments. Ten healthy female swine (Deutsche Landrasse, Sus scrofa Domestica) weighing 44 to $57 \mathrm{~kg}$ were used in this prospective study. The pigs were approximately four months of age. Veterinary inspection on arrival helped to ensure a consistently thorough health status. Animals were housed in pens with a $12 \mathrm{~h}$-day-night cycle and access to drinking water ad libitum. Twelve hours prior to the experiment, animals were set on nil per os, except for drinking water access.

\section{Animal instrumentation}

Weighing of the animals was performed before experiments were started. General anesthesia was induced by intramuscular injection of $4 \mathrm{mg} / \mathrm{kg}$ azaperone (Stresnil, Janssen-Cilag $\mathrm{GmbH}$, Neuss, Germany), followed by intravenous injection of $15 \mathrm{mg} / \mathrm{kg}$ sodium pentobarbital (Narcoren, Boehringer Ingelheim Vetmedica GmbH, Ingelheim am Rhein, Germany). Anesthesia was preserved by continuous intravenous sodium pentobarbital infusion at a rate of $4 \mathrm{mg} / \mathrm{kg} / \mathrm{h}$. Placed in supine position, animals were orotracheally intubated and legs were carefully fixed in an extended position. Animals were ventilated with an inspiratory oxygen fraction of 0.3 (Servo Ventilator 300A; Siemens AG, Munich, Germany). Tidal volume was set to $10 \mathrm{ml} / \mathrm{kg}$ and respiratory rate was adjusted to keep the end-tidal carbon dioxide tension within a physiologic range $(35 \pm 4 \mathrm{mmHg})$. Continuous five lead electrocardiogram (ECG) and pulse oximetry were performed. Convective heating was used to maintain swine's body temperature at $38.0 \pm 0.5^{\circ} \mathrm{C}$ during preparation (Warm Touch 5200; Tyco Healthcare, Pleasanton, CA, USA). At completion of the protocol, anesthesia was stopped and animals were weaned from the ventilator.

\section{Hemodynamic monitoring}

Arterial blood pressure was obtained using a fluid-filled catheter (Vygon, Ecquen, France), that was placed into the left femoral artery. For pulmonary artery pressure and cardiac output $\left(\mathrm{CO}_{\mathrm{TD}}\right)$ measurements by thermodilution, a Swan-Ganz catheter (744HF75; Edwards Lifesciences, Irvine, CA, USA) was flow-directed into the pulmonary artery and connected to a cardiac output monitor (Vigilance; Edward Lifesciences). When hemodynamic monitoring had been fully established, animals were left untreated for approximately thirty minutes. After this stabilization period, comprehensive baseline measurements were obtained.

\section{Echocardiography}

Echocardiography was performed using a GE Vivid E9 system equipped with a 6VT-D $(3.0-8.0 \mathrm{MHz})$ TEE probe (GE Vingmed Ultrasound AS, Horten, Norway). Frames were recorded ECG-synchronized from at least three consecutive cardiac cycles and stored on a hard disk. Two experienced and certificated sonographers performed the TEEs and the offline data analysis. TEE studies and chamber quantification were performed according to the guidelines of the American Society of echocardiography and the European association of cardiovascular imaging [2,21]. The data analysis including myocardial work estimation was carried out using the commercially available software EchoPAC (Version 202; GE Vingmed Ultrasound AS, Horten, Norway). EchoPAC provides validated assessment of LV myocardial work indices. To our knowledge, measurements for RV myocardial work indices with EchoPAC are not validated by GE healthcare or by any other research group yet.

For placement of the TEE-probe, a custom-made bite guard was inserted into the pig's snout. The probe was protected by an ultrasound gel filled probe cover. Before probe introduction, we lubricated the coat's outer surface with ultrasound gel. To avoid advancement into the porcine pharyngeal pouch, the tongue was lifted with a spatula, and the TEE probe was carefully inserted from the right side of the pig's pharynx, applying minimum force [6, 22].

\section{TEE views}

In general, anteflexion and lateroflexion of the probe was needed for unaffected imaging. Small adjustments in rotation, depth and flection of the probe were made in every animal to adapt to the individual anatomy. At $0-20^{\circ}$ array 
position with slight retroflexion, the echocardiographic evaluation was started with a mid-esophageal modified fourchamber (4CH) view (Fig. 1a). In addition to the standard human four-chamber view, this plane allows for imaging of the pulmonary artery as well. From the mid-esophageal position, we assessed the ventricles in further views by modifying the transducer rotation: A two-chamber $(2 \mathrm{CH})$ view was generated at a beam rotation of $60-90^{\circ}$ (Fig. 1b). To obtain the $2 \mathrm{CH}$ view a deep esophageal probe placement and even sometimes a transgastric position was needed. Further beam rotation to $100-130^{\circ}$ resulted in a mid-esophageal threechamber-view, showing a long axis (LAX) of the left ventricle and right ventricular outflow tract (RVOT) (Fig. 1c). An upper-esophageal view with $4-6 \mathrm{~cm}$ withdrawal of the probe from the mid-esophageal position and plane rotation of the transducer between $40^{\circ}$ and $70^{\circ}$ visualized the pulmonary and the aortic valve in a LAX (Fig. 1d). Further withdrawal of the probe resulted in a LAX in- and outflow view of the $\mathrm{RV}$ including the RV free wall.

\section{Chamber quantification}

$\mathrm{LV}$ dimensions were measured in the mid-esophageal $4 \mathrm{CH}$ view and deep esophageal $2 \mathrm{CH}$ view in systole and diastole.
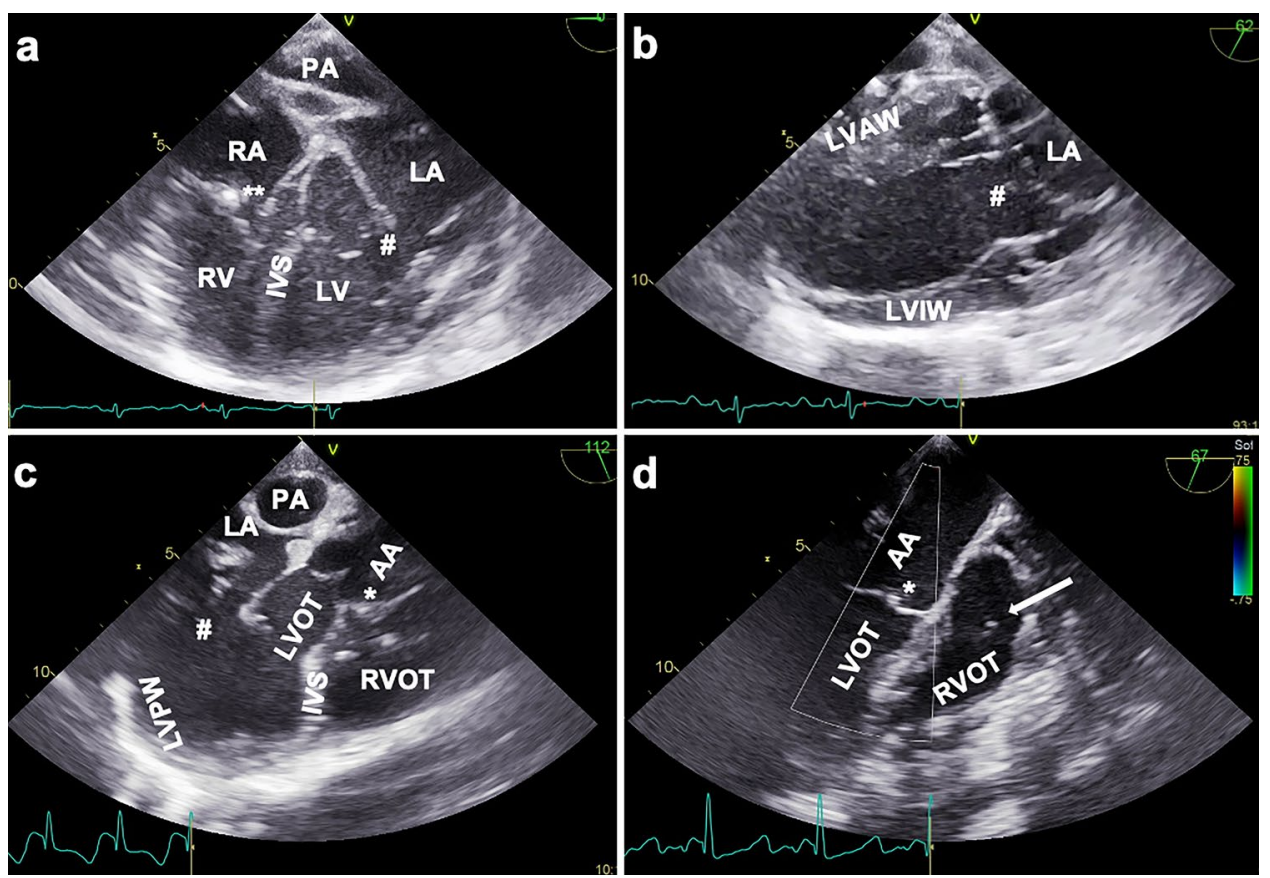

Fig. 1 Representative TEE views in swine. a TEE mid-esophageal modified four-chamber view, array at $0^{\circ} . R A$ right atrium, $* *$ tricuspid valve, $R V$ right ventricle, $I V S$ interventricular septum, $P A$ pulmonary artery, $L A$ left atrium, \# mitral valve, $L V$ left ventricle. b TEE transgastric two-chamber view, array at $62^{\circ} . L V A W$ left ventricular anterior wall, $L V I W$ left ventricular inferior wall, $L A$ left atrium, \# mitral valve. c TEE mid-esophageal three-chamber view, showing the left ventricle in a long axis, array at $112^{\circ}$. $P A$ pulmonary artery,
Left ventricular end-diastolic and end-systolic volumes (LVEDV, LVESV) were determined using biplane method of disk summation, also known as Simpson's biplane method as recommended by ASE and EACVI [1].

$\mathrm{RV}$ basal diameter $\mathrm{D}_{1}$, mid diameter $\mathrm{D}_{2}$ and longitudinal diameter $\mathrm{D}_{3}$ were measured in the mid-esophageal modified $4 \mathrm{CH}$ view.

\section{Systolic ventricular function}

Ejection fraction (EF) was calculated using the modified biplane Simpson's method.

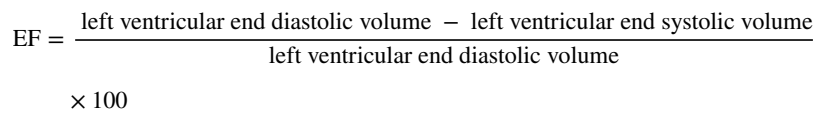

Cardiac output $\left(\mathrm{CO}_{\mathrm{vol}}\right)$ was calculated using the volumetric Simpson's method. For calculation of the stroke volume index, body surface area (BSA) was calculated from the body weight using the following equation $[3,23]$ :

$$
\text { BSA }=0.0734 \times \text { body weight }{ }^{0.656}
$$

$L A$ left atrium, \# mitral valve, $L V P W$ left ventricular posterior wall, LVOT left ventricular outflow tract, * aortic valve, $A A$ ascending aorta, RVOT right ventricular outflow tract, IVS interventricular septum. d TEE upper-oesophageal view of ascending aorta and aortic valve, array at $67^{\circ} . L V O T$ left ventricular outflow tract, * aortic valve, $A A$ ascending aorta, RVOT right ventricular outflow tract, arrow pulmonary valve 
Color Doppler was applied in $4 \mathrm{CH}$ view to exclude any valvular pathologies.

Right ventricular fractional area change (RVFAC) and the tricuspid annular systolic velocity (TASV) were determined as indices of right ventricular function in the $4 \mathrm{CH}$ view. Endocardial right ventricular borders were traced in end-systole and end-diastole to obtain the corresponding RV areas. Subsequently, RVFAC was calculated [24]:

$\mathrm{RVFAC}=\frac{\mathrm{RV} \text { end diastolic area }-\mathrm{RV} \text { end systolic area }}{\mathrm{RV} \text { end diastolic area }} \times 100$

TASV was captured in $4 \mathrm{CH}$ views at the intersection of the $\mathrm{RV}$ free wall and the anterior leaflet of the tricuspid valve using tissue Doppler (Fig. 2a) [25].

\section{Hemodynamics}

Pulsed wave Doppler (PWD) was applied in the LAX-views of the pulmonary and the aortic valve to obtain the corresponding velocity time integrals (VTI) from the left (LVOT) and right (RVOT) ventricular outflow tract (Fig. 2b and c). Upper esophageal LAX-views provided sufficient angle alignment between blood flow and Doppler ultrasound transducer. Ultrasound derived Cardiac output $\left(\mathrm{CO}_{\mathrm{PWD}}\right)$ was computed using the following equations [26].

$\mathrm{CO}_{\mathrm{PWD} \text { pulmonary }}=\mathrm{RVOT} \mathrm{VTI} \times$ cross - sectional area $_{\mathrm{RVOT}} \times$ heart rate

$\mathrm{CO}_{\mathrm{PWD} \text { aortic }}=$ LVOT VTI $\times$ cross - sectional area $\mathrm{LVOT}_{\mathrm{LVO}} \times$ heart rate

$\mathrm{CO}$ was divided by the heart rate to obtain the pulmonary and aortic stroke volume (SV).

\section{Deformation imaging and myocardial work analysis}

Myocardial deformation imaging using 2DSTE offers an advanced LV quantification beyond ejection fraction (EF) [19]. However, 2DSTE is load-dependent and therefore changes in afterload can affect the diagnostic value of 2DSTE [19]. In 2012, Russell et al. [18] introduced a novel non-invasive method to calculate global myocardial work index (GWI) using pressure-strain loops. This novel non-invasive myocardial work parameter takes afterload as well as deformation into account and is therefore considered superior to 2DSTE as it offers additional insights into myocardial function [27].

Before performing 2DSTE, aortic and mitral valve opening and closure time were determined by pulsed-Doppler from the mid-esophageal LAX view. Deformation imaging of the LV was applied in the mid-esophageal modified
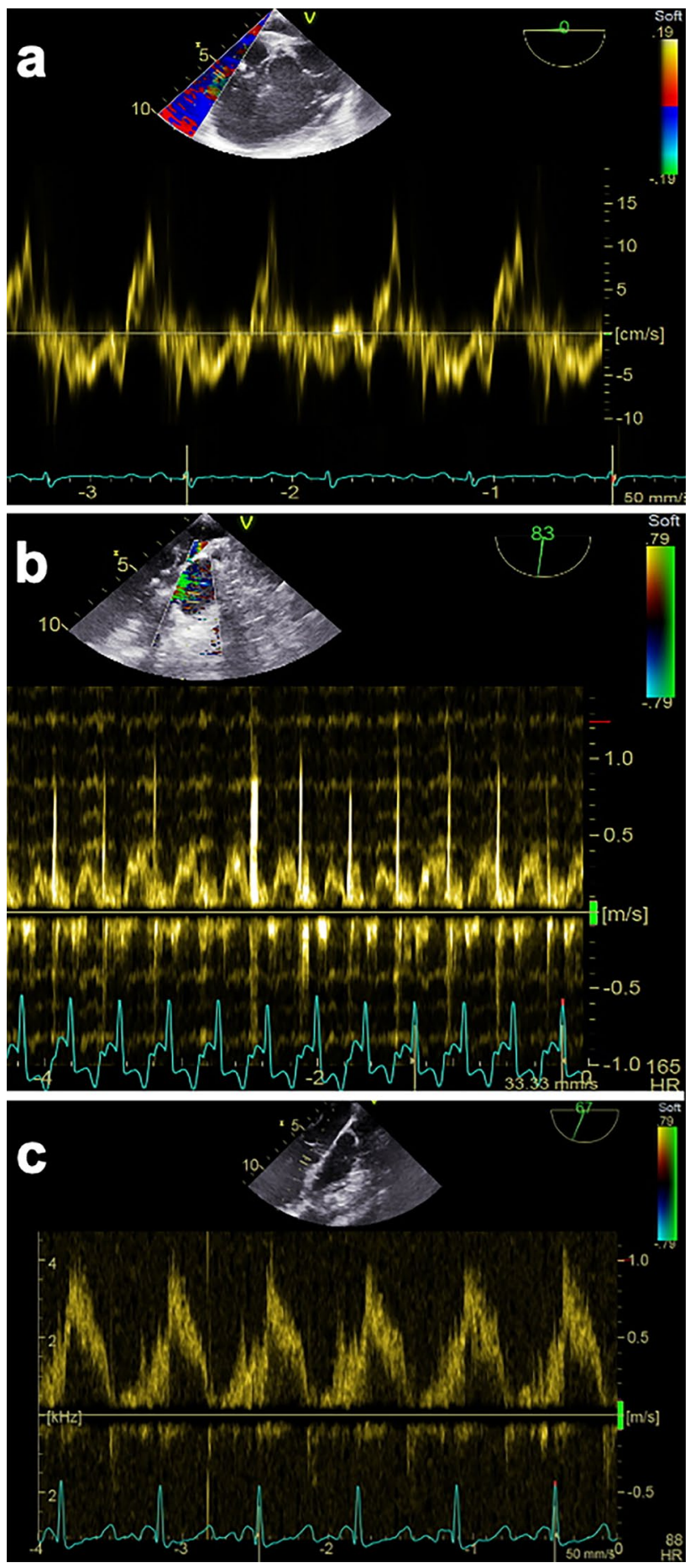

Fig. 2 Representative TEE pulsed wave Doppler and tissue Doppler measurements. a Tissue Doppler beam aligned with the lateral tricuspidal annulus to measure tricuspidal annular plane systolic velocity (TASV). b TEE pulsed wave Doppler measurement in the right ventricular outflow tract. c TEE pulsed wave Doppler measurement in the left ventricular outflow tract 

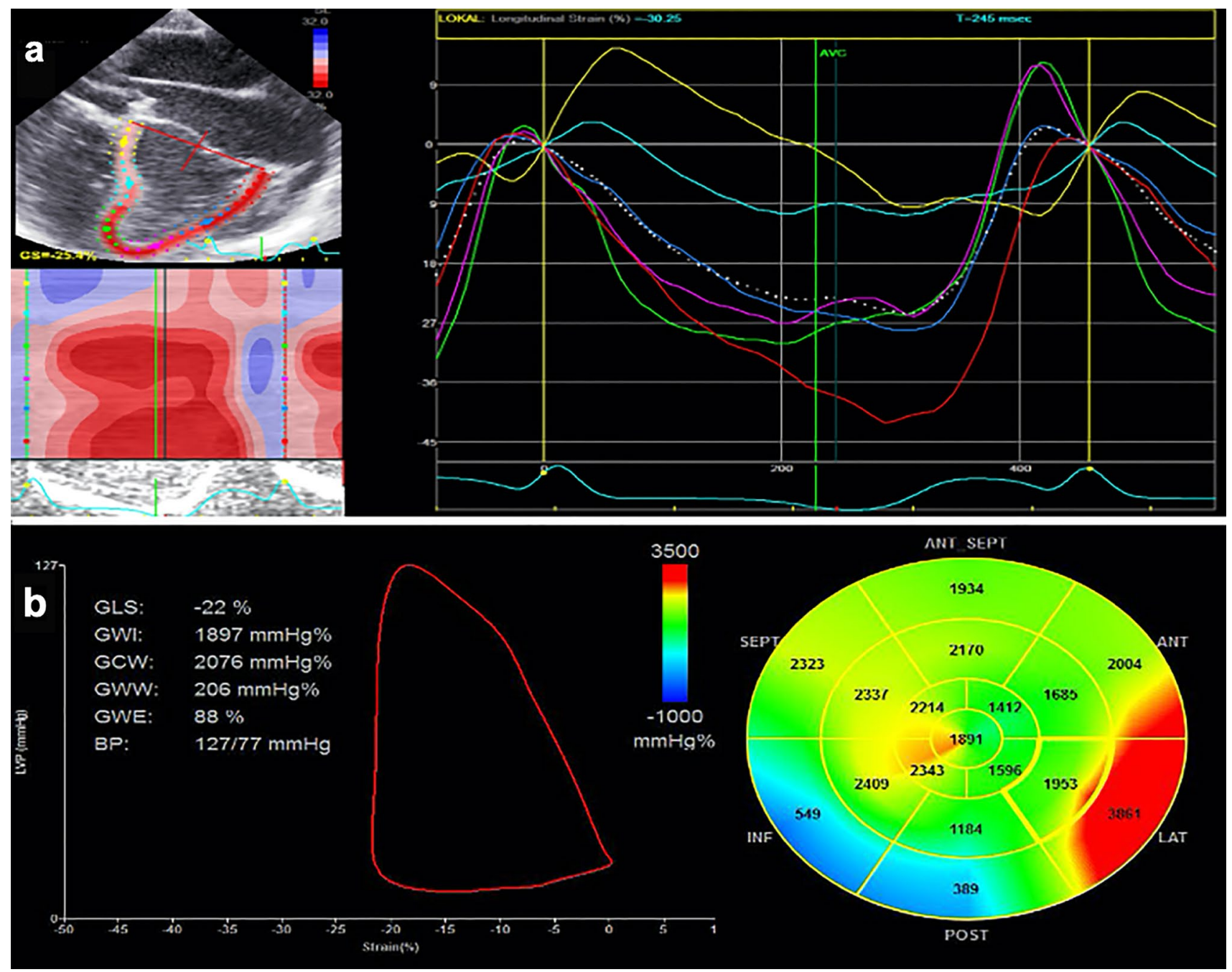

Fig. 3 Exemplary demonstration of speckle trasssscking and myocardial work analysis. a Analysis of left ventricular peak systolic longitudinal strain obtained from a modified apical 4-chamber view with parametric (color-coded) display of end-systolic strain (left upper panel); M-mode representation of peak systolic strain (left lower panel); and strain-time curves: each colored curve displays a different segmental strain while the dotted white curve represents the aver-

4CH-, LAX- and deep-esophageal 2CH views (Fig. 3a). To perform 2DSTE analysis of the RV we used the $4 \mathrm{CH}$ view for the RV anterior wall (Fig. 4a), the modified upper LAX RV in- and outflow view showing the RV free wall (Fig. 4b) and the mid-esophageal LAX RV view showing the posterior part of the RVOT (Fig. 4c). 2DSTE was used to define LV and RV global longitudinal strain (GLS) [28]. The systolic arterial and pulmonary blood pressure was used to produce pressure-strain loops in EchoPAC (Fig. 3b). Left ventricular pressure is estimated by adjusting a reference pressure curve with a measured blood pressure and with echocardiography derived valvular event timing $[17,18]$. We used the blood pressure derived from a femoral arterial line for the LV, and age peak systolic longitudinal strain (right upper panel). b myocardial work indices obtained from pressure-strain loops with the myocardial work curve and the Bull's eye representing segmental myocardial work indices. $G L S$ global longitudinal strain, GWI global myocardial work index, $G C W$ global constructive work, $G W W$ global wasted work, $G W E$ global effective work, $B P$ blood pressure

the pulmonary artery pressure derived from a Swan-Ganz catheter for the RV.

EchoPAC software creates an adjusted LV pressure curve corresponding to the duration of isovolumic and ejection phases determined by valvular timing events. As described by Russel et al. [17, 18], the area within the PSL delivers the index of the GW. The following parameters can then be calculated:

(1) GWI: the total area of the PSL represents the total work from mitral valve closure to mitral valve opening.

(2) Constructive MW (GCW): work of the LV, which contributes to the LV ejection during systole. Constructive 

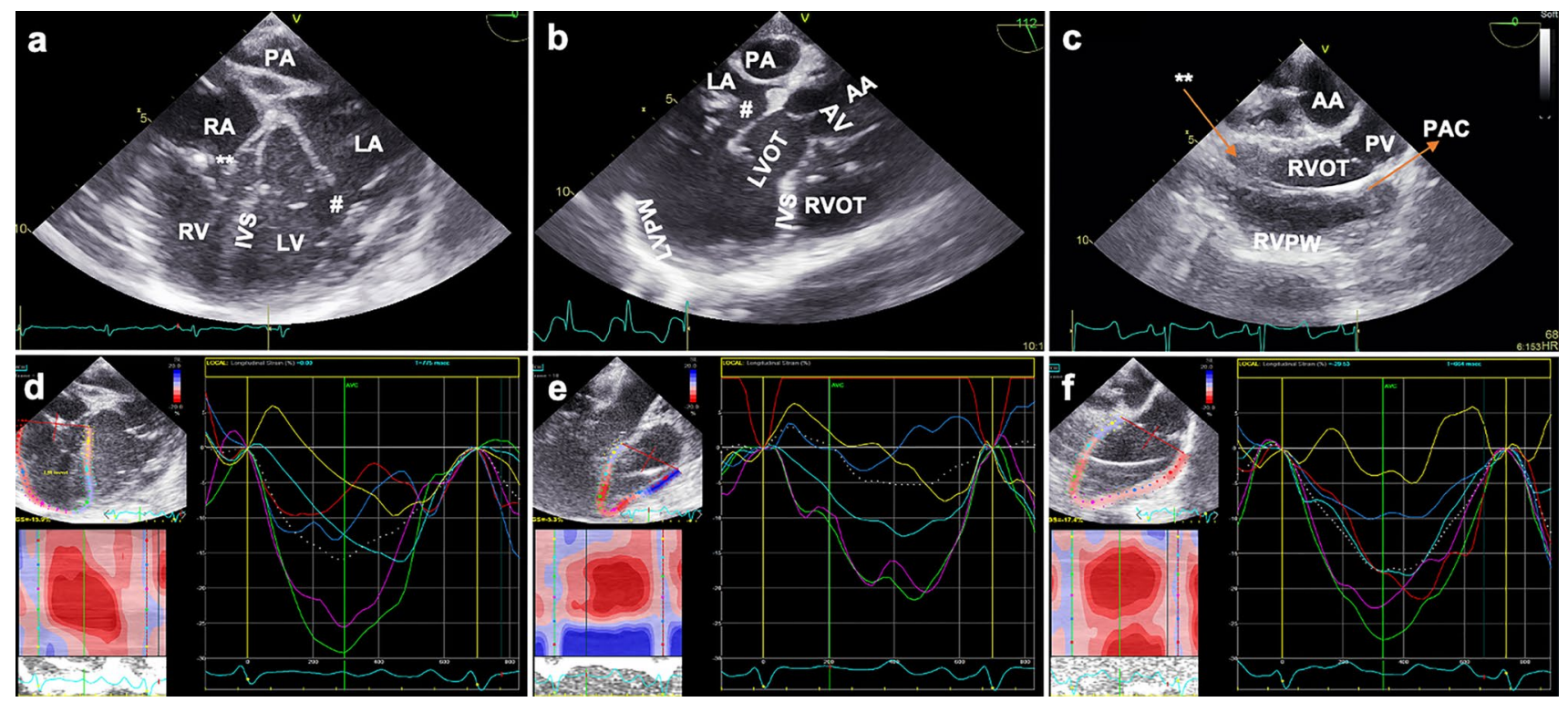

Fig. 4 Right ventricular myocardial work assessment. a $4 \mathrm{CH}$ view for the $\mathrm{RV}$ anterior wall at $0^{\circ} . R A$ right atrium, $* *$ tricuspid valve, $R V$ right ventricle, IVS interventricular septum, $P A$ pulmonary artery, $L A$ left atrium, \# mitral valve, $L V$ left ventricle. b modified upper LAX RV in- and outflow view showing the RV free wall at $112^{\circ}$. $P A$ pulmonary artery, $L A$ left atrium, \# mitral valve, $A V$ aortic valve, $A A$ ascending aorta, LVOT left ventricular outflow tract, IVS interventricular septum, RVOT right ventricular outflow tract, $L V P W$ left ventricular posterior wall. c mid-esophageal LAX RVOT view showing the posterior part of the RVOT at $0^{\circ}$. $A A$ ascending aorta, RVOT right ventricular outflow tract, $R V P W$ right ventricular posterior wall, ** tricuspid valve, $P A C$ pulmonary artery catheter, $P V$ pulmonary

MW is defined as shortening of the myocytes during systole. GCW = positive Work during systole + negative work during isovolumic relaxation.

(3) Wasted MW (GWW): Work of the LV that does not contribute to the LV-ejection. Wasted MW is defined as lengthening of the myocytes (instead of shortening) during systole, which further intensifies the shortening during the isovolumic relaxation phase. GWW = negative work during systole + positive work during isovolumic relaxation.

(4) MW Efficiency (GWE): is the fraction of constructive MW to total work and calculated by GCW/(GCW + GWW) $[17,18]$.

\section{Analysis}

We used SPSS statistics Version 26 (IBM, Armonk, NY, USA) for data analysis. Normal distribution was confirmed using Kolmogorov-Smirnov test. Normal distributed measurements are expressed as mean \pm standard deviation. If normal distribution was not present, data are expressed as median followed by range in brackets. To test for correlation between $\mathrm{CO}$ derived from indwelling catheters and valve. d peak systolic longitudinal strain analysis of the anterior right ventricular obtained from a $4 \mathrm{CH}$ view (corresponding to panel a). e peak systolic longitudinal strain analysis of the right ventricular free wall obtained from a modified upper LAX RV in- and outflow (corresponding to panel $b$ ). f peak systolic longitudinal strain analysis of the right ventricular posterior part of the RVOT (corresponding to panel c). Panels (d-f): parametric (color-coded) display of end-systolic strain (left upper panel); M-mode representation of peak systolic strain (left lower panel); and strain-time curves: each colored curve displays a different segmental strain while the dotted white curve represents the average peak systolic longitudinal strain (right panel).

echocardiographic measurements, we ran a Pearson correlation test. A $p$ value smaller than 0.05 was considered significant.

\section{Results}

We carried out a comprehensive TEE examination in 10 healthy female swine with a median body weight of 46.1 $(43.6-56.7) \mathrm{kg}$. Study acquisition took on average between 20 and 30 min per animal. No adverse events concerning TEE probe insertion or TEE-study acquisition in general were observed. All the above-described views and structures could be visualized in each animal. No preexisting cardiac conditions were found in any of the examined animals.

\section{Chamber quantification}

LVEDV was $70.7 \pm 4.4 \mathrm{ml}$ and LVESV was $32.6 \pm 3.8 \mathrm{ml}$. RV basal diameter $D_{1}$ was $3.3 \pm 0.4 \mathrm{~cm}$, mid diameter $\mathrm{D}_{2}$ was $2.5(2.4-3.1) \mathrm{cm}$ and longitudinal diameter $\mathrm{D}_{3}$ was 4.4 $\pm 0.2 \mathrm{~cm}$. 


\section{Systolic ventricular function}

RVFAC was $44 \pm 6 \%$ and TASV was $13.1 \pm 1.8 \mathrm{~cm} / \mathrm{s}$ General physiological parameters as well as TEE data about ventricular size and function are presented in Table $1 . \mathrm{LV}$ SV was $70.5 \pm 5.9 \mathrm{ml}$ and the biplane EF was $63.1 \pm 7.1 \%$. BSA adjusted SV index was $75.6 \pm 7.2 \mathrm{ml} / \mathrm{m}^{2}$ for $\mathrm{LV}$ and $76.7 \pm 7.8 \mathrm{ml} / \mathrm{m}^{2}$ for $\mathrm{RV}$.

\section{Deformation imaging and myocardial work analysis}

GLS was $-21.3 \pm 3.9 \%$ for LV and $-15.4 \pm 2.5 \%$ for RV. GWI was 1885 (1281-2121) $\mathrm{mmHg} * \%$ for $\mathrm{LV}$ and $297 \pm 62$ $\mathrm{mmHg} * \%$ for RV. GCW was $2100 \pm 421 \mathrm{mmHg} * \%$ for LV and $322 \pm 84 \mathrm{mmHg} * \%$ for RV. Those values are comparable to values found in humans (Table 2). LV GWW was $360 \pm 151 \mathrm{mmHg} * \%$ and $59 \pm 11 \mathrm{mmHg} * \%$ for RV. GWE was $82.6 \pm 4 \%$ for the LV and 83(72-88) \% for the RV. LV GWW was about four-fold higher in swine then in men translating into a lower LV GWE in swine than in men (82.6 $\pm 4 \%)$ in swine vs. 96 (94-97)\% in men.

\section{Hemodynamics}

Mean heart rate during the examinations was $92 \pm 13$ beats per minute. Mean arterial blood pressure was $128 \pm 17$ $\mathrm{mmHg}$ systolic and $68 \pm 6 \mathrm{mmHg}$ diastolic. Mean pulmonary artery pressure was $26 \pm 3 \mathrm{mmHg}$ systolic and $13 \pm 3$ $\mathrm{mmHg}$ diastolic. PW-Doppler estimated LV SV was $70.5 \pm$ $5.9 \mathrm{ml}$ and SV indexed to BSA was $75.6 \pm 7.2 \mathrm{ml} / \mathrm{m}^{2}$ for LV and $76.7 \pm 7.8 \mathrm{ml} / \mathrm{m}^{2}$ for RV.

$\mathrm{CO}$ was evaluated by three different methods. Thermodilution-method derived $\mathrm{CO}_{\mathrm{TD}}$ was $7.0 \pm 1.1 \mathrm{l} / \mathrm{min}$. PWD derived pulmonary $\mathrm{CO}_{\mathrm{PWD}}$ was $7.0 \pm 1.2 \mathrm{1} / \mathrm{min}$ and $\mathrm{PWD}$ derived aortic output was $6.8 \pm 1.1 \mathrm{l} / \mathrm{min}$. $\mathrm{CO}_{\mathrm{vol}}$ calculated by the volumetric Simpson's method was $5.1 \pm 1.1 \mathrm{l} / \mathrm{min}$.

A linear regression analysis and pearson's correlation were performed to compare the estimated $\mathrm{CO}$ between the three methods (Fig. 5). Invasively measured $\mathrm{CO}_{\mathrm{TD}}$ correlated

Table 1 General data and TEE findings in swine and humans.

\begin{tabular}{|c|c|c|c|c|}
\hline Parameter & Swine mean & $\mathrm{SD} /$ (range) & Mean human & Human SD \\
\hline Weight (kg) & 46.1 & $(43.6-56.7)$ & & \\
\hline Body surface area $\left(\mathrm{m}^{2}\right)$ & 0.91 & $(0.88-1.04)$ & & \\
\hline Heart rate $\left(\mathrm{min}^{-1}\right)$ & 92 & \pm 13 & & \\
\hline Swan-Ganz catheter derived cardiac output $\left(1 / \mathrm{min}^{-1}\right)$ & 7.03 & \pm 1.1 & & \\
\hline \multicolumn{5}{|l|}{ Right ventricular dimensions and function } \\
\hline Right ventricle basal diameter $\mathrm{D}_{1}(\mathrm{~cm})$ & 3.3 & \pm 0.4 & $3.3^{\mathrm{a}}$ & $\pm 0.4^{\mathrm{a}}$ \\
\hline Right ventricle mid diameter $\mathrm{D}_{2}(\mathrm{~cm})$ & 2.5 & $(2.4-3.1)$ & $2.7^{\mathrm{a}}$ & $\pm 0.4^{\mathrm{a}}$ \\
\hline Right ventricle longitudinal diameter $\mathrm{D}_{3}(\mathrm{~cm})$ & 4.4 & \pm 0.2 & $7.1^{\mathrm{a}}$ & $\pm 0.6^{\mathrm{a}}$ \\
\hline Right ventricular end diastolic area $\left(\mathrm{cm}^{2}\right)$ & 10.5 & \pm 1.6 & & \\
\hline Right ventricular end systolic area $\left(\mathrm{cm}^{2}\right)$ & 5.9 & \pm 0.9 & & \\
\hline Right ventricular fractional area change (\%) & 44 & \pm 6 & $49^{\mathrm{a}}$ & $\pm 7^{\mathrm{a}}$ \\
\hline Tricuspid annular systolic velocity $(\mathrm{cm} / \mathrm{s})$ & 13.1 & \pm 1.8 & $14.1^{\mathrm{a}}$ & $\pm 2.3^{\mathrm{a}}$ \\
\hline Pulmonal cardiac output (1/min) & 7.0 & \pm 1.2 & & \\
\hline Pulmonal stroke volume (ml) & 71.6 & \pm 7.3 & & \\
\hline Pulmonal stroke volume index $\left(\mathrm{ml} / \mathrm{m}^{2}\right)$ & 76.7 & \pm 7.8 & & \\
\hline \multicolumn{5}{|l|}{ Left ventricular dimensions and function } \\
\hline Left ventricular end diastolic volume (ml) & 70.7 & \pm 4.4 & $76^{\mathrm{b}}$ & $\pm 15^{\mathrm{b}}$ \\
\hline Left ventricular end systolic volume (ml) & 32.6 & \pm 3.8 & $28^{\mathrm{b}}$ & $\pm 7^{\mathrm{b}}$ \\
\hline Left ventricular ejection fraction $(\%)$ & 63.1 & \pm 7.1 & $64^{\mathrm{b}}$ & $\pm 5^{\mathrm{b}}$ \\
\hline Aortic cardiac output (1/min) & 6.8 & \pm 1.1 & & \\
\hline Aortic cardiac index $\left(1 / \mathrm{m}^{2}\right)$ & 7.35 & \pm 1.2 & $4.41^{\mathrm{c}}$ & \\
\hline Aortic stroke volume (ml) & 70.5 & \pm 5.9 & & \\
\hline Aortic stroke volume index $\left(\mathrm{ml} / \mathrm{m}^{2}\right)$ & 75.6 & \pm 7.2 & & \\
\hline
\end{tabular}

Values are expressed as mean \pm standard deviation. Data in brackets represent median and range of not normal distributed data. Selected human values are presented for reference

${ }^{\text {a } D a t a}$ reported in [31]

${ }^{b}$ Data reported in [1], values are presented for a female group

${ }^{\mathrm{c}}$ Data reported in [48] 
Table 2 TEE derived myocardial work values in pigs and humans

\begin{tabular}{|c|c|c|c|c|}
\hline parameter & Swine mean & $\mathrm{SD} /$ (range) & Human mean & Human SD/(range) \\
\hline \multicolumn{5}{|l|}{ Left ventricular mechanics } \\
\hline Systolic blood pressure $(\mathrm{mmHg})$ & 128 & \pm 17 & $116^{\mathrm{a}}$ & $\pm 12^{\mathrm{a}}$ \\
\hline Diastolic blood pressure (mmHg) & 68 & \pm 6 & $73^{\mathrm{a}}$ & $\pm 8^{\mathrm{a}}$ \\
\hline Global longitudinal strain (\%) & -21.3 & \pm 3.9 & $-21.2^{b}$ & $\pm 2.4^{\mathrm{b}}$ \\
\hline Global work index $(\mathrm{mmHg} * \%)$ & 1885 & $(1281-2121)$ & $1896^{\mathrm{a}}$ & $\pm 308^{\mathrm{a}}$ \\
\hline Global constructive work (mmHg*\%) & 2100 & \pm 421 & $2232^{\mathrm{a}}$ & $\pm 331^{\mathrm{a}}$ \\
\hline Global wasted work $(\mathrm{mmHg} * \%)$ & 360 & \pm 151 & $78.5^{\mathrm{a}}$ & $(53-122.2)^{\mathrm{a}}$ \\
\hline Global myocardial work efficiency (\%) & 82.6 & \pm 4 & $96^{\mathrm{a}}$ & $(94-97)^{\mathrm{a}}$ \\
\hline \multicolumn{5}{|l|}{ Right ventricular mechanics } \\
\hline Systolic pulmonal arterial pressure $(\mathrm{mmHg})$ & 26 & \pm 3 & & \\
\hline Diastolic pulmonal arterial pressure $(\mathrm{mmHg})$ & 13 & \pm 3 & & \\
\hline Global longitudinal strain (\%) & -15.4 & \pm 2.5 & & \\
\hline Global work index $(\mathrm{mmHg} * \%)$ & 297 & \pm 62 & & \\
\hline Global constructive work $(\mathrm{mmHg} * \%)$ & 322 & \pm 84 & & \\
\hline Global wasted work $(\mathrm{mmHg} * \%)$ & 59 & \pm 11 & & \\
\hline Global myocardial work efficiency (\%) & 83 & $(62-88)$ & & \\
\hline
\end{tabular}

Data are expressed as mean \pm standard deviation. Data in brackets represent median and range of not normal distributed data

${ }^{\text {a }}$ Data reported in [44]

${ }^{\mathrm{b}}$ Data reported in [1]

well with both the PWD derived $\mathrm{CO}_{\mathrm{PWD}}(r=0.812, p=$ $0.004)$ and the volumetric estimated $\mathrm{CO}_{\mathrm{vol}}(r=0.672, p=$ 0.032 ).

\section{Discussion}

The study at hand is unique in its capability to demonstrate both feasibility and reliability of sophisticated TEE based measurements in anesthetized swine. To the best of our knowledge, this is the first trial to evaluate LV and RV myocardial function and mechanics using the novel noneinvasive myocardial work estimation method by Russel and colleagues in this setting. Moreover, our data not only complement porcine standard values that have been determined by others [29, 30], but furthermore adds novel insight into myocardial work of both ventricles in swine.

These observations are significant due to the importance of porcine animal models of cardiovascular disease. Swine are frequently chosen as model-organism for cardiovascular disease due to their close resemblance of human anatomy and physiology [3-5]. More specifically, our findings show that LV GLS in swine is comparable to LV GLS in man. LV GWI and GCW were also comparable to standard values derived from human data. Conversely, global wasted work was found to be four times higher in swine with 360 $\mathrm{mmHg} * \%$ vs. $78.5 \mathrm{mmHg} * \%$ in men. One possible explanation is that our measurements were carried out in anesthetized swine, while human reference values were derived from TTE in awake healthy subjects. Hence, anesthesia drugs and positive pressure ventilation appear to have shifted the myocardial wasted work ratio towards negative work in our setting.

Our RV data about TASV and the RVFAC appear to resemble findings in humans $[1,31]$. In terms of dimensions, it is noticeable that RV basal and mid diameters $\mathrm{D}_{1}$ and $\mathrm{D}_{2}$ were comparable in swine and humans, while the longitudinal diameter $\mathrm{D}_{3}$ was shorter in swine [31]. By and large, the measured LV parameters were comparable to that in humans. The EF was similar, while $\mathrm{CO}$ was found to be higher in swine than in humans. However, other studies in swine found similar values regarding $\mathrm{CO}$ and $\mathrm{RV} / \mathrm{LV}$ volumes [15, 29-32]. As swine are known to compensate for a low blood hemoglobin level with a high $\mathrm{CO}$ in order to achieve a sufficient oxygen tissue supply [33], the higher CO may be a result of structural and physiological differences between the species.

Comparing our data to the porcine TEE study of Huenges et al. we noticed a similar EF in conjunction with half the $\mathrm{CO}$ found in our experiments [14], despite being estimated in equally sized animals. These differences in $\mathrm{CO}$ values may be based on differences in the anesthesia regimen. Furthermore, $\mathrm{CO}$ is calculated using an LVOT cross sectional area that is prone to misestimation.

Validation of our echo-obtained CO data with an invasively measured reference method [26] vastly improved the credibility of our results. We observed a strong positive correlation of cardiac output measured by thermodilution 

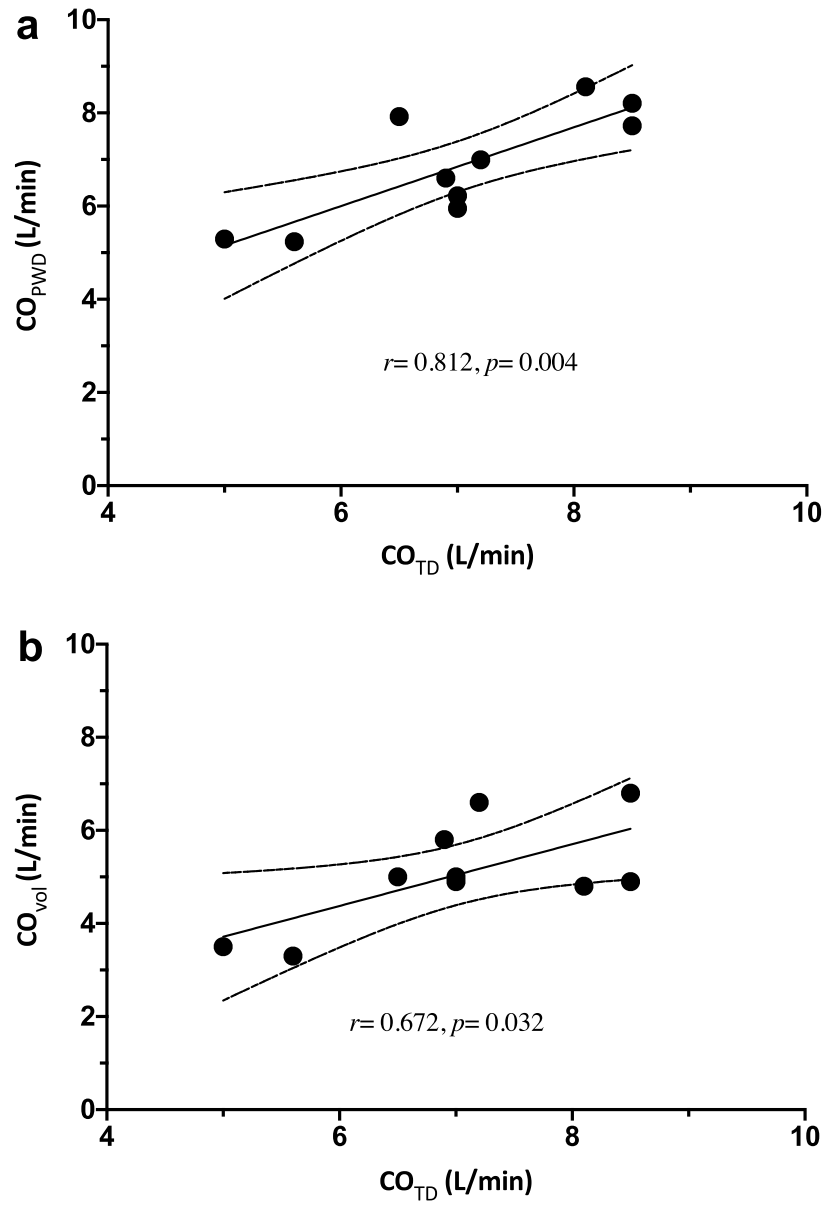

Fig. 5 Correlation plot of thermodilution, pulsed wave Doppler and volumetric derived cardiac output. Invasively obtained cardiac output measured by pulmonary artery catheter correlated well with TEE measurements. a Comparison of $\mathrm{CO}_{\mathrm{TD}}$ and $\mathrm{CO}_{\mathrm{PWD}} \mathbf{b}$ Comparison of $\mathrm{CO}_{\mathrm{TD}}$ and $\mathrm{CO}_{\mathrm{vol}} \cdot \mathrm{CO}_{\mathrm{TD}}=$ cardiac output determined using thermodilution via a Swan-Ganz catheter, $\mathrm{CO}_{\mathrm{PWD}}=$ Ultrasound derived Cardiac output using pulsed wave Doppler, $\mathrm{CO}_{\mathrm{vol}}=$ Ultrasound derived Cardiac output using volumetric calculations. Dotted lines represent 95\%-confidence interval

with PWD derived values. This correlation implies a good accuracy of our echocardiographic measurements. The correlation between volumetric estimated $\mathrm{CO}_{\mathrm{vol}}$ and the thermodilution $\mathrm{CO}_{\mathrm{TD}}$ was good as well, but lower than between $\mathrm{CO}_{\mathrm{PWD}}$ and $\mathrm{CO}_{\mathrm{vol}}$. One possible explanation for the discrepancy between $\mathrm{CO}_{\mathrm{PWD}}$ and $\mathrm{CO}_{\mathrm{vol}}$ is the difficulty to obtain the correct anatomical 2-CH view and the 4-CH leading to underestimated values due to measurements in foreshortened views.

Certain limitations apply to the results gained in our study. First, study results were obtained in a fairly small sample size of 10 animals. However, choosing a small sample size is particularly desirable from an ethical perspective wherever results are valid. Second, TEE measurements were performed in swine anaesthetized with pentobarbital.
Previous research revealed conflicting data on pentobarbital effects on hemodynamics as compared to awake animals [34-37]. However, all known anesthetics have significant impact on hemodynamic properties [38], and acquisition of TEE-studies in swine is next to impossible without sedation. Third, imaging the apex is tremendously difficult in swine. Attention was paid to avoid foreshortening of the ventricles in echocardiography and ante- and retroflexion of the tip was used to find the appropriate plane of the heart. However, foreshortening of the ventricle might have caused an underestimation of the concerning ventricular volumes. 3D-TEE is believed to overcome these limitations and would probably have led to a more precise calculation of derived parameters [26]. Other important limitations are imposed by the RV myocardial work indices used here. To the best of our knowledge, no specific software for non-invasive RV myocardial work assessment is commercially available at this time. Furthermore, peer-reviewed publications on comparisons of TEE-derived pressure-strain measurements versus invasive pressure-volume loops are lacking. In our analysis, we used commercially available LV software provided by GE. Therefore, results from our RV myocardial work studies must be interpreted with caution. Furthermore, image quality was limited in a few cases. These limitations are due to specific anatomical features of swine in which the main bronchus may interleave between heart and esophagus [15, 16]. Another limitation concerning the MW measurements is a possible difference between central arterial systolic pressure and femoral arterial systolic pressure. Central pressure might have been lower than peripheral pressure due to pressure augmentation. Heart rate variability and arrhythmia, with significant beat-to-beat variability affect the accuracy and reliability of 2DSTE assessments with the viability of MW estimation. However, significant arrhythmia was not observed in any of the examined animals. Animals were scheduled to undergo a separate experimental protocol following the echocardiographic assessments described in this manuscript. As this protocol included weaning of the pigs from the ventilator, invasive measures were kept to an absolute minimum in order to reduce the risk for procedure related adverse events. Hence, we failed to determine left (LVP) and right ventricular pressure (RVP) that would have allowed for plotting of true pressure-volume loops. However, doing so will be an additional asset that will be incorporated into future experimental protocols.

Compared to TTE, TEE offers significant advantages in swine: The close anatomic relation of the esophagus to the heart enables high resolution images of the heart. TEE can be applied in the closed chest without significant trauma. This can be paramount in chronic animal experiments. In addition, TEE may be favorable in open-chest models, as it allows for echocardiographic assessment without interfering with surgical interventions. If longitudinal strain values 
are to be measured, TEE might also be the superior choice over TTE, as apical views may not be achievable using transthoracic echocardiography. However, for the acquisition of longitudinal strain calculation, apical planes are critical [12, 39].

In general, an important constraint in interpretation of conventional strain data is the load dependency [19, 28]. Afterload-dependence of the strain must be considered especially in settings in which large fluctuations of the afterload are likely to occur. We used a method described by Russel et al. that is more robust to afterload changes than strain with regards to described systolic function. This method computes work with strain imaging and an estimated left ventricular pressure $[19,20]$. The calculated myocardial work reflects myocardial oxygen-consumption and metabolic rate $[18,40,41]$. This methodology has been employed in human clinical research before [19, 20, 42-44]. Transferring this method to animal experiments opens up new options for translational research: For example, the impact of new drugs on myocardial work can be studied semi-invasive by TEE in chronic experiments. This method might also be used in the context of cardiac stimulation research. Cardiac resynchronization therapy has been evaluated in humans using Russel's method by analyzing the wasted myocardial work before and after implantation of a resynchronization device $[45,46]$. The efficacy of novel cardiac stimulation systems might therefore be studied with TEE in a porcine model of heart failure in the future. Furthermore, consideration of the performed myocardial work is crucial in the evaluation of ventricular assist devices, as they are supposed to reduce heart work. Research on left ventricular assist devices with the help of echocardiographic assessed myocardial work is likely to bring new insights into this technology, especially as the strategies presented here may be employed to monitor RV function that is known to be critical to assist device function [47].

\section{Conclusions}

TEE offers sufficient morphological, functional and hemodynamic assessment of the heart in swine. The non-invasive GWI may offer an improved assessment of myocardial contractility and mechanics. The established baseline values will inform design and validation of future studies in translational cardiovascular research.

Funding Open Access funding enabled and organized by Projekt DEAL. This work was supported by the German Research Foundation (Deutsche Forschungsgemeinschaft, DFG) [Grant Number DE 1685/31]. The sponsor was not involved in study design; in the collection, analysis and interpretation of data; in the writing of the report; and in the decision to submit the article for publication.
Data availability The authors declare that they have full control of all primary data and that they agree to allow the journal to review their data if requested.

\section{Compliance with ethical standards}

Conflicts of interest The authors declare that they have no known competing financial interests or personal relationships that could have appeared to influence the work reported in this paper.

Open Access This article is licensed under a Creative Commons Attribution 4.0 International License, which permits use, sharing, adaptation, distribution and reproduction in any medium or format, as long as you give appropriate credit to the original author(s) and the source, provide a link to the Creative Commons licence, and indicate if changes were made. The images or other third party material in this article are included in the article's Creative Commons licence, unless indicated otherwise in a credit line to the material. If material is not included in the article's Creative Commons licence and your intended use is not permitted by statutory regulation or exceeds the permitted use, you will need to obtain permission directly from the copyright holder. To view a copy of this licence, visit http://creativecommons.org/licenses/by/4.0/.

\section{References}

1. Lang RM, Badano LP, Mor-Avi V, Afilalo J, Armstrong A, Ernande L, et al (2015) Recommendations for cardiac chamber quantification by echocardiography in adults: an update from the American Society of Echocardiography and the European Association of Cardiovascular Imaging. J Am Soc Echocardiogr 28(1):1-39 e14.

2. Hahn RT, Abraham T, Adams MS, Bruce CJ, Glas KE, Lang RM et al (2013) Guidelines for performing a comprehensive transesophageal echocardiographic examination: recommendations from the American Society of Echocardiography and the Society of Cardiovascular Anesthesiologists. J Am Soc Echocardiogr 26(9):921-64

3. Swindle MM, Makin A, Herron AJ, Clubb FJ Jr, Frazier KS (2012) Swine as models in biomedical research and toxicology testing. Vet Pathol 49(2):344-56

4. Crick SJ, Sheppard MN, Ho SY, Gebstein L, Anderson RH (1998) Anatomy of the pig heart: comparisons with normal human cardiac structure. J Anat 193 (Pt 1)(Pt 1):105-19.

5. Lelovas PP, Kostomitsopoulos NG, Xanthos TT (2014) A comparative anatomic and physiologic overview of the porcine heart. J Am Assoc Lab Anim Sci 53(5):432-8

6. Ellenbroek GH, van Hout GP, Timmers L, Doevendans PA, Pasterkamp G, Hoefer IE (2016) Primary outcome assessment in a pig model of acute myocardial infarction. J Vis Exp 116:54021

7. Ballo H, Tarkia M, Haavisto M, Stark C, Strandberg M, Vahasilta $T$ et al (2019) Determinants of myocardial strain in experimental chronic myocardial infarction. Ultrasound Med Biol 45(2):568-78

8. Ruan W, Lu L, Zhang Q, Cao M, Zhu ZB, Wang LJ et al (2009) Serial assessment of left ventricular remodeling and function by echo-tissue Doppler imaging after myocardial infarction in streptozotocin-induced diabetic swine. J Am Soc Echocardiogr 22(5):530-6

9. Fang J, Wang R, Liu H, Su Y, Chen J, Han X et al (2020) Transapical septal myectomy in the beating heart via a minimally invasive approach: a feasibility study in swine. Interact Cardiovasc Thorac Surg 30(2):303-311

10. Loger K, Lutter G, Huenges K, Frank D, Gross J, Cremer J et al (2018) Transcatheter mitral valve implantation: supra-annular 
and subvalvular fixation techniques. Eur J Cardiothorac Surg 54(6):1013-21

11. Kerut EK, Valina CM, Luka T, Pinkernell K, Delafontaine P, Alt EU (2004) Technique and imaging for transthoracic echocardiography of the laboratory pig. Echocardiography 21(5):439-42

12. Koudstaal S, Jansen of Lorkeers S, Gho JM, van Hout GP, Jansen MS, Grundeman PF, et al (2014) Myocardial infarction and functional outcome assessment in pigs. J Vis $\operatorname{Exp}(86): 51269$

13. Weidemann F, Jamal F, Sutherland GR, Claus P, Kowalski M, Hatle L et al (2002) Myocardial function defined by strain rate and strain during alterations in inotropic states and heart rate. Am J Physiol Heart Circ Physiol 283(2):H792-9

14. Huenges K, Pokorny S, Berndt R, Cremer J, Lutter G (2017) Transesophageal echocardiography in swine: establishment of a baseline. Ultrasound Med Biol 43(5):974-80

15. Ren JF, Schwartzman D, Lighty GW Jr, Menz VV, Michele JJ, Li $\mathrm{KS}$ et al (1997) Multiplane transesophageal and intracardiac echocardiography in large swine: imaging technique, normal values, and research applications. Echocardiography 14(2):135-48

16. Sündermann Simon H, Cesarovic Nikola, Falk Volkmar, Bettex Dominique (2016) Two- and three-dimensional transoesophageal echocardiography in large swine used as model for transcatheter heart valve therapies: standard planes and values. Interact CardioVasc Thorac Surg 22:580-6

17. Russell K, Eriksen M, Aaberge L, Wilhelmsen N, Skulstad H, Gjesdal O et al (2013) Assessment of wasted myocardial work: a novel method to quantify energy loss due to uncoordinated left ventricular contractions. Am J Physiol Heart Circ Physiol 305(7):H996-1003

18. Russell K, Eriksen M, Aaberge L, Wilhelmsen N, Skulstad H, Remme EW et al (2012) A novel clinical method for quantification of regional left ventricular pressure-strain loop area: a noninvasive index of myocardial work. Eur Heart J 33(6):724-33

19. Boe E, Russell K, Eek C, Eriksen M, Remme EW, Smiseth OA et al (2015) Non-invasive myocardial work index identifies acute coronary occlusion in patients with non-ST-segment elevation-acute coronary syndrome. Eur Heart J Cardiovasc Imag 16(11):1247-55

20. Edwards NFA, Scalia GM, Shiino K, Sabapathy S, Anderson B, Chamberlain R et al (2019) Global myocardial work is superior to global longitudinal strain to predict significant coronary artery disease in patients with normal left ventricular function and wall motion. J Am Soc Echocardiogr 32(8):947-57

21. Flachskampf FA, Badano L, Daniel WG, Feneck RO, Fox KF, Fraser AG et al (2010) Recommendations for transoesophageal echocardiography: update 2010. Eur J Echocardiogr 11(7):557-76

22. Seaman DL, de la Mora Levy J, Gostout CJ, Rajan E, Herman L, Knipschield M (2007) An animal training model for endoscopic treatment of Zenker's diverticulum. Gastrointest Endosc 65(7):1050-3

23. Kelley KW, Curtis SE, Marzan GT, Karara HM, Anderson CR (1973) Body surface area of female swine. J Anim Sci 36(5):927-30

24. Jones N, Burns AT, Prior DL (2019) Echocardiographic assessment of the right ventricle-state of the art. Heart Lung Circ 28(9):1339-50

25. Meluzin J, Spinarova L, Bakala J, Toman J, Krejci J, Hude P et al (2001) Pulsed doppler tissue imaging of the velocity of tricuspid annular systolic motion; a new, rapid, and non-invasive method of evaluating right ventricular systolic function. Eur Heart J 22(4):340-8

26. Shimada YJ, Ishikawa K, Kawase Y, Ladage D, Tilemann L, Shiota $\mathrm{T}$ et al (2012) Comparison of left ventricular stroke volume assessment by two- and three-dimensional echocardiography in a swine model of acute myocardial infarction validated by thermodilution method. Echocardiography 29(9):1091-5
27. Chan J, Edwards NFA, Khandheria BK, Shiino K, Sabapathy S, Anderson B et al (2019) A new approach to assess myocardial work by non-invasive left ventricular pressure-strain relations in hypertension and dilated cardiomyopathy. Eur Heart J Cardiovasc Imag 20(1):31-9

28. Mor-Avi V, Lang RM, Badano LP, Belohlavek M, Cardim NM, Derumeaux G et al (2011) Current and evolving echocardiographic techniques for the quantitative evaluation of cardiac mechanics: ASE/EAE consensus statement on methodology and indications endorsed by the Japanese Society of Echocardiography. J Am Soc Echocardiogr 24(3):277-313

29. van Essen GJ, Te Lintel HM, Sorop O, Heinonen I, van der Velden J, Merkus D et al (2018) Cardiovascular function of modern pigs does not comply with allometric scaling laws. Sci Rep. 8(1):792

30. Stam K, Chelu RG, van der Velde N, van Duin R, Wielopolski P, Nieman K et al (2019) Validation of 4D flow CMR against simultaneous invasive hemodynamic measurements: a swine study. Int J Cardiovasc Imag 35(6):1111-8

31. Venkatachalam S, Wu G, Ahmad M (2017) Echocardiographic assessment of the right ventricle in the current era: application in clinical practice. Echocardiography 34(12):1930-47

32. Meybohm P, Gruenewald M, Renner J, Maracke M, Rossee S, Hocker J et al (2011) Assessment of left ventricular systolic function during acute myocardial ischemia: a comparison of transpulmonary thermodilution and transesophageal echocardiography. Minerva Anestesiol 77(2):132-41

33. Hannon JPBC, Wade CE (1990) Normal physiological values for conscious pigs used in biomedical research. Lab Anim Sci 40(3):293-8

34. Barlow G, Knott DH (1964) Hemodynamic alterations after 30 minutes of pentobarbital sodium anesthesia in dogs. Am J Physiol 207:764-6

35. Li C, Dai J, Wu F, Zhang H (2019) Impacts of different anesthetic agents on left ventricular systolic function in mice assessed by echocardiography. Physiol Res 68(3):365-74

36. Sawyer DC, Lumb WV, Stone HL (1971) Cardiovascular effects of halothane, methoxyflurane, pentobarbital, and thiamylal. J Appl Physiol 30(1):36-43

37. Stein AB, Tiwari $\mathrm{S}$, Thomas $\mathrm{P}$, Hunt $\mathrm{G}$, Levent $\mathrm{C}$, Stoddard MF et al (2007) Effects of anesthesia on echocardiographic assessment of left ventricular structure and function in rats. Basic Res Cardiol 102(1):28-41

38. Goodchild CS, Serrao JM (2015) Propofol-induced cardiovascular depression: science and art. Br J Anaesth 115(4):641-2

39. Jamal F, Kukulski T, Strotmann J, Szilard M, D'Hooge J, Bijnens B et al (2001) Quantification of the spectrum of changes in regional myocardial function during acute ischemia in closed chest pigs: an ultrasonic strain rate and strain study. J Am Soc Echocardiogr 14(9):874-84

40. Boe E, Skulstad H, Smiseth OA (2019) Myocardial work by echocardiography: a novel method ready for clinical testing. Eur Heart J Cardiovasc Imaging. 20(1):18-20

41. Suga H (1990) Ventricular energetics. Physiol Rev 70(2):247-77

42. Galli E, John-Matthwes B, Rousseau C, Schnell F, Leclercq C, Donal E (2019) Echocardiographic reference ranges for myocardial work in healthy subjects: a preliminary study. Echocardiography 36(10): 1814-24

43. Hubert A, Le Rolle V, Leclercq C, Galli E, Samset E, Casset C et al (2018) Estimation of myocardial work from pressure-strain loops analysis: an experimental evaluation. Eur Heart J Cardiovasc Imag 19(12):1372-9

44. Manganaro R, Marchetta S, Dulgheru R, Ilardi F, Sugimoto T, Robinet $S$ et al (2019) Echocardiographic reference ranges for normal non-invasive myocardial work indices: results from the EACVI NORRE study. Eur Heart J Cardiovasc Imag 20(5):582-90 
45. Galli E, Leclercq C, Fournet M, Hubert A, Bernard A, Smiseth OA et al (2018) Value of myocardial work estimation in the prediction of response to cardiac resynchronization therapy. J Am Soc Echocardiogr 31(2):220-30

46. Zhu M, Chen H, Fulati Z, Liu Y, Su Y, Shu X (2019) The value of left ventricular strain-volume loops in predicting response to cardiac resynchronization therapy. Cardiovasc Ultrasound 17(1):3

47. Scalia GM, McCarthy PM, Savage RM, Smedira NG, Thomas JD (2000) Clinical utility of echocardiography in the management of implantable ventricular assist devices. J Am Soc Echocardiogr 13(8):754-63

48. Katori R (1979) Normal cardiac output in relation to age and body size. Tohoku J Exp Med 128(4):377-87

Publisher's Note Springer Nature remains neutral with regard to jurisdictional claims in published maps and institutional affiliations. 